EESTI NSV TEADUSTE AKADEEMIA TOIMETISED.

FOOSIKA * MATEMAATIKA

ИЗВЕСТИЯ АКАДЕМИИ НАУК ЭСТОНСКОИ ССР. ФИЗИКА * МАТЕМАТИКА

PROCEEDINGS OF THE ACADEMY OF SCIENCES OF THE ESTONIAN SSR. PHYSICS * MATHEMATICS

$1987,36,1$

удК 517.986 .2

A. $K O K K$

\title{
ОПИСАНИЕ ГОМОМОРФИЗМОВ ТОПОЛОГИЧЕСКИХ МОДУЛЬ-АЛГЕБР
}

\author{
(Представил А. Хумал)
}

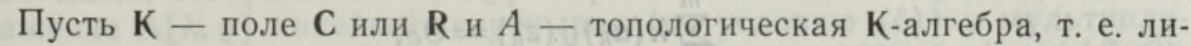
нейное топологическое пространство над К, в котором определено умножение элементов, относительно которого оно является ассоциативной алгеброй, и в топологии которого рассматриваемое умножение элементов (как билинейное отображение $A \times A$ в $A$ ) раздельно непрерывно. В частном случае, когда умножение элементов алгебры $A$ непрерывно в совокупности, $A$ называется топологической К-алгеброй с непрерывным умножением.

Пусть теперь топология алгебры $A$ отделима. Тогда она имеет пополнение $\bar{A}$ (см. напр., [ [ $\left.{ }^{1}\right]$, с. 131 ), являющееся отделимым линейным топологическим пространством, но не обязательно алгеброй (см. $\left[{ }^{2}\right]$, c. 21). В случае, когда $A$ является отделимой топологической К-алгеброй с непрерывным умножением, то умножение элементов алгебры $A$ непрерывно продолжаемо на $\bar{A} \times \bar{A}$ (см. $\left[{ }^{3}\right]$, с. 100$)$. Поэтому в таком случае и пополнение $\bar{A}$ алгебры $A$ является отделимой топологической Қ-алгеброй с непрерывным умножением. При этом алгебра $A$ является топологически изоморфной всюду плотной подалгебре алгебры $\bar{A}$.

Топологическая К-алгебра $B$ называется топологической модульалгеброй относительно алгебры $A$ или, коротко, топологической $(A, \mathbf{K})$ алгеброй, если

1) А есть топологическая К-алгебра,

2) В есть А-бимодуль,

3) модульные умножения алгебры $B($ т. е. отображения $(a, b) \rightarrow a b$ u $(b, a) \rightarrow b a$ произведений $A \times B$ в $B$ и $B \times A$ в $B$ соответственно) раздельно непрерывны

и

4) $a\left(b_{1} b_{2}\right)=\left(a b_{1}\right) b_{2}, \quad\left(b_{1} b_{2}\right) a=b_{1}\left(b_{2} a\right)$ u $b_{1}\left(a b_{2}\right)=\left(b_{1} a\right) b_{2}$ для всех $b_{1}, b_{2} \in B \quad u \quad a \in A$.

При этом, если алгебра $A$ имеет единицу (здесь и всюду в дальнейшем через $e_{A}$ обозначается единица алгебры $A$ ), то $e_{A} b=b=b e_{A}$ для всех $b \in B$, а если алгебра $B$ имеет единицу, то $a e_{B}=e_{B} a$ для всех $a \in A$. Топологическая $(A, \mathbf{K})$-алгебра $B$ называется топологической $(A, \mathbf{K})$ алгеброй с непрерывным умножением, если она является топологической K-алгеброй с непрерывным умножением.

Целью данной статьи является описание гомоморфизмов топологической $(A, \mathbf{K})$-алгебры в топологическую $\mathbf{K}$-алгебру. Для этого, пусть $A$ - топологическая К-алгебра с единицей, $C$ и $D-$ топологические K-алгебры, hom $(A, D)$ - множество всех нетривиальных непрерывных K-гомоморфизмов $A$ в $D$, наделенное слабой тоћологией, $\operatorname{hom} A=$ $=\operatorname{hom}(A, \mathbf{K})$ и $H_{A D C}=\operatorname{hom}(A, C) \times \operatorname{ham}(D, G)$ Пусть далее $\mathbf{N}_{n}=$ $=\{1,2, \ldots, n\}$ для каждого $n \notin \mathbb{N}, \Theta_{\mathrm{A}}$ нулввй элемент алгебры 
$A, B$ - топологическая $(A, \mathbf{K})$-алгебра с единицей, $Z$ - подалгебра алгебры $B$ такая, что $e_{B} \in Z, A Z-A$-подмодуль алгебры $B$, порожденный алгеброй $Z$ и

$$
\Lambda[\lambda ; \varrho]\left(\sum_{k=1}^{n} a_{k} z_{k}\right)=\sum_{k=1}^{n} \lambda\left(a_{k}\right) \varrho\left(z_{k}\right)
$$

для всех $(\lambda, \varrho) \in H_{A Z C}, n \in \mathbf{N}, a_{k} \in A$ и $z_{k} \in Z$ с $k \in \mathbf{N}_{n}$.

В случае, когда отображение $\Lambda \in \operatorname{hom}(A Z, C)$ имеет продолжение на замыкание $\mathrm{cl}_{B} A Z$ подмножества $A Z$ в топологии алгебры $B$, то это продолжение будем обозначать через $\bar{\Lambda}$. Кроме того, через $S_{A Z C}$ будем обозначать подмножество тех пар $(\lambda, \varrho) \in H_{A Z C}$, для которых выполнены условия

(а) если $m \in \mathbf{N}, a_{k} \in A$ и $z_{k} \in Z \quad$ длякаждого $k \in \mathbf{N}_{m}$ суть такие, что $a_{1} z_{1}+a_{2} z_{2}+\ldots+a_{m} z_{m}=\Theta_{A Z}$, TO

$$
\sum_{k=1}^{m} \lambda\left(a_{k}\right) \varrho\left(z_{k}\right)=\Theta_{C},
$$

( $\beta) ~ \lambda(a) \varrho(z)=\varrho(z) \lambda(a)$ при всех $a \in A \quad u z \in Z$,

( $\gamma) \lambda\left(e_{A}\right)=\mathrm{Q}\left(e_{B}\right)$

$u$

(8) отображение $\Lambda[\lambda, \varrho]$ непрерывно на $A Z$.

Основные результаты данной статьи следующие.

Т ео р м а 1. Пусть $A$ - топологнческая К-алгебра $c$ единицей, C - топологическая К-алгебра с непрерывным умножением, $B$ - топологическая $(A, \mathbf{K})$-алгебра с единицей и $Z$ - подалгебра алгебры $B$ такая, что $e_{B} \in Z$. Если

$u$

(a) $a z=z a$ при всех $a \in A$ u $z \in Z$

(б) множество hom $(A Z, C)$ непусто,

то каждый гомоморфизм $\Lambda \in \operatorname{hom}(A Z, C)$ определяет элемент $(\lambda, \mathrm{Q}) \in$

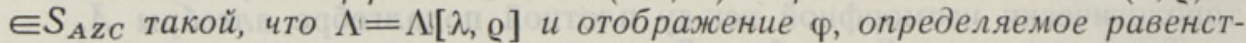
вом $\varphi(\lambda, \varrho)=\Lambda[\lambda, \varrho]$ для всех $(\lambda, \varrho) \in S_{A Z C}$, является гомеоморфизмом пространств $S_{A z c} u$ hom $(A Z, C)$.

Т еорем а 2. В предположениях теоремы 1 пусть алгебра $C$ полна и отделима, умножение в алгебре $B$ непрерывно и пусть выполнено условие

(в) подмодуль $A Z$ алгебры В всюду плотен в $B$.

Тогда каждый гомоморфизм $\Lambda \in \operatorname{hom}(B, C)$ определяет элемент $(\lambda, \varrho) \in$ $\in S_{A z c}$ такой, что $\Lambda=\overline{\Lambda[\lambda, \varrho]}$, и отображение $\Phi$, определяемое равенством $\Phi(\lambda, \varrho)=\overline{\Lambda[\lambda, \varrho]}$ при всех $(\lambda, \varrho) \in S_{A z c}$, является биекцией $S_{A z c} н а$ hom $(B, C)$, обратное отображение которой непрерывно на $\operatorname{hom}(B, C)$.

В частном случае, когда выполнено и условие

(г) пространство hom $(A Z, C)$ локально равностепенно непрерывно, то Ф является гомеоморфизмом пространств $S_{A z c} u \operatorname{hom}(B, C)$.

Т е о р е а 3. Пусть $A$ - топологическая К-алгебра $c$ единицей, $C$ - полная отделимая топологическая К-алгебра с непрерывным умножением, $B$ - отделимая топологическая $(A, \mathbf{K})$-алгебра с единицей и непрерывным умножением, а $Z$ - такая подалгебра алгебры $B$, что $e_{B} \in Z$. Если выполнены условия (а), (б), (в) $и$ (г), то пространства $S_{A Z C} u$ hom $(\bar{B}, C)$ гомеоморфны.

1. Доказательство теоремы 1. Ясно, что подмодуль $A Z$ образует (по условию (а)) подалгебру алгебры $B$. Пусть $\Lambda \in \operatorname{hom}(A Z, C)$. Положим $\varrho_{\Lambda}=\Lambda \mid Z$ (т.е. $\varrho_{\Lambda}$ является сужением отображения $\Lambda$ на $Z$ ) и 
$\lambda_{\Lambda}(a)=\Lambda\left(a e_{B}\right)$

для каждого $a \in A$. Поскольку гомоморфнзм $\Lambda$ нетривиален на $A Z$, то и отображения $\lambda_{\Lambda}$ и $\varrho_{\Lambda}$ нетривиальны на $A$ и $Z$ соответственно, ибо $\lambda_{\Lambda}\left(e_{A}\right)=\varrho_{\Lambda}\left(\bullet_{B}\right)=\Lambda\left(e_{B}\right)$. Нетрудно заметить, что отображения $\lambda_{\Lambda}$ и $\mathrm{Q}_{\Lambda}$ непрерывны, линейны и мультипликативны на $A$ и $Z$ соответственно. Кроме того, пара $\left(\lambda_{\Lambda} ; \varrho_{\Lambda}\right)$ удовлетворяет условиям $(\beta)$ и $(\gamma)$ (по условию (а)). Так как

$$
\Lambda\left(\sum_{k=1}^{n} a_{k} z_{k}\right)=\sum_{k=1}^{n} \lambda_{\Lambda}\left(a_{k}\right) \varrho_{\Lambda}\left(z_{k}\right)
$$

при всех $n \in \mathbf{N}, a_{i} \in A$ и $z_{i} \in Z$ с $i \in \mathbf{N}_{n}$, то $\Lambda=\Lambda\left[\lambda_{\Lambda}, \varrho_{\Lambda}\right]$ и выполнены условия $(\alpha)$ и $(\delta)$. Значит, $\left(\lambda_{\Lambda}, \varrho_{\Lambda}\right) \in S_{A Z G}$.

Пусть теперь $(\lambda, \varrho) \in S_{A Z C}$. Поскольку $\varrho \in \operatorname{hom}(Z, C)$, то $\varrho\left(e_{B}\right) \neq$ $\neq \Theta_{C}$. Поэтому $\Lambda[\lambda, \varrho]\left(e_{B}\right)=\lambda\left(e_{A}\right) \varrho\left(e_{B}\right) \neq \Theta_{C} \quad$ (по условию $\left.(\gamma)\right)$. Следовательно, отображение $\Lambda[\lambda$, , ] нетривиально на $A Z$. Кроме того, отображение $\Lambda[\lambda, \varrho]$ линейно, непрерывно (по условию $(\delta)$ ) и мультипликативно (по условию $(\beta))$ на $A Z$. Таким образом, $\Lambda[\lambda, \varrho] \in \operatorname{hom}(A Z, C)$.

Пусть далее $\left(\lambda_{1} ; \varrho_{1}\right),\left(\lambda_{2}, \varrho_{2}\right) \in S_{A Z C}$ и $\left(\lambda_{1}, \varrho_{1}\right) \neq\left(\lambda_{2}, \varrho_{2}\right)$. Тогда справедливо либо $\Lambda\left[\lambda_{1}, \varrho_{1}\right]\left(a_{0} e_{B}\right)=\lambda_{1}\left(a_{0}\right) \neq \lambda_{2}\left(a_{0}\right)=\Lambda\left[\lambda_{2}, \varrho_{2}\right]\left(a_{0} e_{B}\right)$, либо $\Lambda\left[\lambda_{1}, \varrho_{1}\right]\left(z_{0}\right)=\varrho_{1}\left(z_{0}\right) \neq \varrho_{2}\left(z_{0}\right)=\Lambda\left[\lambda_{2}, \varrho_{2}\right]\left(z_{0}\right)$ для некоторых $a_{0} \in A$ и $z_{0} \in Z$ (по условию $\left.(\gamma)\right)$. Поэтому $\Lambda\left[\lambda_{1}, \varrho_{1}\right] \neq \Lambda\left[\lambda_{2}, \varrho_{2}\right]$. Следовательно, $\varphi$ (определяемое равенством $\varphi(\lambda, \varrho)=\Lambda[\lambda, \varrho]$ при всех $\left.(\lambda, \varrho) \in S_{A Z C}\right)$ отображает множество $S_{A Z C}$ взаимно однозначно на $\operatorname{hom}(A Z, C)$.

Покажем теперь непрерывность отображения чр. Для этого, пусть $\left(\lambda_{0}, \varrho_{0}\right) \in S_{A Z c}$ и $O_{0}\left(\Lambda_{0}\right)$ - любая окрестность элемента $\Lambda_{0}=\varphi\left(\lambda_{0}, \varrho_{0}\right)$ в топологии пространства $\operatorname{hom}(A Z, C)$. Тогда существуют число $r \in \mathbf{N}$ и для каждого $k \in \mathbf{N}_{r}$ число $m(k) \in \mathbf{N}$, элементы $a_{k, 1}, a_{k, 2}, \ldots, a_{k, m(k)} \in A$ и $z_{k, 1}, z_{k, 2}, \ldots, z_{k, m(k)} \in Z$ и окрестность нуля $U_{k}$ алгебры $C$ такие, что

$$
\bigcap_{k=1}^{r}\left\{\Lambda \in \operatorname{hom}(A Z, C):\left(\Lambda-\Lambda_{0}\right)\left(d_{k}\right) \in U_{k}\right\} \subseteq O_{0}\left(\Lambda_{0}\right),
$$

где $\quad d_{k}=a_{k, 1} z_{k, 1}+a_{k, 2} z_{k, 2}+\ldots+a_{k, m(k)} z_{k, m(k)}$. Пусть $x_{k, i}=\Lambda_{0}\left(a_{k, i} z_{k, i}\right) \quad$ и $y_{k}=\Lambda_{0}\left(d_{k}\right)$ для всех $k \in \mathbf{N}_{r}$ и $i \in \mathbf{N}_{m(k)}$. Тогда в алгебре $C$ найдутся окрестности $T_{k, i}$ элементов $x_{k, i}$ такие, что $T_{k, 1}+T_{k, 2}+\ldots+T_{k, m(k)} \subset y_{k}+$ $+U_{k}$ при всех $k \in \mathbf{N}_{r}$. В силу непрерывности умножения в алгебре $C$ найдутся, в свою очередь, окрестности $S_{k, i}$ элементов $\lambda_{0}\left(a_{k, i}\right)$ и окрестности $R_{k, i}$ элементов @o $\left(z_{k, i}\right)$ такие, что $S_{k, i} R_{k, i} \subset T_{k, i}$ при всех $k \in \mathbf{N}_{r}$ и $i \in \mathbf{N}_{m(k)}$.

Пусть теперь $S_{k, i}=\lambda_{0}\left(a_{k, i}\right)+V_{k, i}$ и $R_{k, i}=\varrho_{0}\left(z_{k, i}\right)+W_{k, i}$, где $V_{k, i}$ и $W_{k, i}$ - окрестности нуля алгебры $C$ для всех $k \in \mathbf{N}_{r}$ и $i \in \mathbf{N}_{m(k)}$. Кроме того, пусть

и

$$
O_{1}=\bigcap_{k=1}^{r} \bigcap_{i=1}^{m(k)}\left\{\lambda \in \operatorname{hom}(A, C):\left(\lambda-\lambda_{0}\right)\left(a_{k, i}\right) \in V_{k, i}\right\}
$$

$$
O_{2}=\bigcap_{k=1}^{r} \bigcap_{i=1}^{m(k)}\left\{\varrho \in \operatorname{hom}(Z, C):\left(\varrho-\varrho_{0}\right)\left(z_{k, i}\right) \in W_{k, i}\right\} \text {. }
$$

Тогда из $(\lambda, \varrho) \in O_{3}=\left(O_{1} \times O_{2}\right) \cap S_{A Z c}$ следует, что $\lambda\left(a_{k, i}\right) \in S_{k, i}$ и $\varrho\left(z_{k, i}\right) \in R_{k, i}$. Поэтому $\lambda\left(a_{k, i}\right) \varrho\left(z_{k, i}\right) \in T_{k, i}$ при всех $(\lambda ; \varrho) \in O_{3}, k \in \mathbf{N}_{r}$ и $i \in \mathbf{N}_{m(k)}$. Таким образом, элемент $c_{k}=\varphi(\lambda, \varrho)\left(d_{k}\right)$ принадлежит окрестности $y_{k}+U_{k}$, а элемент $\left(\varphi(\lambda, \varrho)-\Lambda_{0}\right)\left(d_{k}\right)=c_{k}-y_{k}-$ окрестности $U_{k}$ для всех $k \in \mathbf{N}_{r}$. Значит, $\varphi(\lambda, \varrho) \in O_{0}\left(\Lambda_{0}\right)$ при всех $(\lambda, \varrho) \in O_{3}$. Поскольку $\mathrm{O}_{3}$ является окрестностью точки $\left(\lambda_{0}, \mathrm{Q}_{0}\right)$ в топологии, индуцированной на $S_{A Z C}$ пространством $H_{A Z C}$, то биекция $\varphi$ непрерывна в любой точке $\left(\lambda_{0}, Q_{0}\right) \in S_{A z C}$. 
Покажем теперь и непрерывность отображения $\varphi^{-1}$. Для этого пусть $\Lambda_{1} \in \operatorname{hom}(A Z, C)$ и $O$ - любая окрестность элемента $\left(\lambda_{1}, \varrho_{1}\right)=\varphi^{-1}\left(\Lambda_{1}\right)$ в топологии пространства $S_{A Z C}$. Тогда в алгебре $C$ существуют окрестности нуля $V_{1}, V_{2}, \ldots, V_{n}, W_{1}, W_{2}, \ldots, W_{m}$ и элементы $a_{1}, a_{2}, \ldots, a_{n} \in A$ и $z_{1}, z_{2}, \ldots, z_{m} \in Z$ такие, что $\left(O_{4} \times O_{5}\right) \cap S_{A Z C} \subset O$, где

$$
O_{4}=\bigcap_{k=1}^{n}\left\{\lambda \in \operatorname{hom}(A, C):\left(\lambda-\lambda_{1}\right)\left(a_{k}\right) \in V_{k}\right\}
$$

и

$$
O_{5}=\bigcap_{\gamma=1}^{m}\left\{\varrho \in \operatorname{hom}(Z, C):\left(\varrho-\varrho_{1}\right)\left(z_{v}\right) \in W_{\nu}\right\} .
$$

Пусть далее

и

$$
O_{6}=\bigcap_{k=1}^{n}\left\{\Lambda \in \operatorname{hom}(A Z, C):\left(\Lambda-\Lambda_{1}\right)\left(a_{k} e_{B}\right) \in V_{k}\right\}
$$

$$
O_{7}=\bigcap_{v=1}^{m}\left\{\Lambda \in \operatorname{hom}(A Z, C):\left(\Lambda-\Lambda_{1}\right)\left(z_{v}\right) \in W_{\gamma}\right\}
$$

Тогда $O_{1}\left(\Lambda_{1}\right)=O_{6} \cap O_{7}$ является окрестностью элемента $\Lambda_{1}$ в топологии пространства hom $(A Z, C)$. Пусть теперь $\Lambda \in O_{1}\left(\Lambda_{1}\right)$. Тогда, по изложенному выше, $\Lambda=\Lambda[\lambda, \varrho]$ для некоторого элемента $(\lambda, \varrho) \in S_{A z c}$. Так как $\left(\lambda-\lambda_{1}\right)\left(a_{k}\right)=\left(\Lambda-\Lambda_{1}\right)\left(a_{k} e_{B}\right) \in V_{k}$ для всех $k \in \mathbf{N}_{n} \quad$ и $\left(\varrho-\varrho_{1}\right)\left(z_{v}\right)=$ $=\left(\Lambda-\Lambda_{1}\right)\left(z_{v}\right) \in W_{v}$ для всех $v \in \mathbf{N}_{m}$, то $\varphi^{-1}(\Lambda)=(\lambda, \varrho) \in O$. Итак, отображение $\varphi^{-1}$ непрерывно в любой точке $\Lambda_{1} \in \operatorname{hom}(A Z, C)$. Следовательно, отображение $\varphi$ является гомеоморфизмом пространств $S_{A Z C}$ и hom $(A Z, C)$.

\section{2. Для доказательства теоремы 2 нам понадобится}

Пр едлож ен и е * 1. Пусть D-топологическая К-алгебра с непрерывным умножением, $E$ - всюду плотная подалгебра алгебры $D u$ H - полная отделимая топологическая К-алгебра с непрерывным умножением такие, что множество $\operatorname{hom}(E, H)$ непусто. Тогда каждый гомоморфизм $\omega \in \operatorname{hom}(E, H)$ продолжим до гомоморфизма $\bar{\omega} \in \operatorname{hom}(D, H)$ u отображение $\mu$, определяемое равенством $\mu(\varrho)=\varrho \mid E$ для всех $\varrho \in$ $\in \operatorname{hom}(D, H)$, является непрерывным и взаимно однозначным отображением пространства $\operatorname{hom}(D, H)$ на $\operatorname{hom}(E, H)$. В частном случае, когда пространство $\operatorname{hom}(E, H)$ локально равностепенно непрерывно, $\mu$ является гомеоморфизмом пространств $\operatorname{hom}(D, H) u \operatorname{hom}(E, H)$.

Доказательство. Пусть $\mathrm{Q}$ - любой элемент пространства $\operatorname{hom}(D, H)$. Поскольку $E$ есть всюду плотная подалгебра алгебры $D$, то $\mathrm{Q} \mid E € \operatorname{hom}(E, H)$. Чтобы показать сюръективность отображения $\mu$, возьмем любой элемент $\omega \in \operatorname{hom}(E, H)$. Тогда существует нетривиальное непрерывное линейное отображение $\bar{\omega}: D \rightarrow H$ такое, что $\omega=\bar{\omega} \mid E$ (см. $\left[{ }^{1}\right]$, c. 129). Оказывается, что отображение $\bar{\omega}$ является и мультипликативным на $D$. Действительно, пусть $d_{1}, d_{2} \in D$ и $O-$ любая окрестность нуля алгебры $H$. Поскольку умножение в алгебре $H$ непрерывно, то в ней существует закругленная окрестность нуля $O_{1}$ такая, что $O_{1}^{2}+O_{1} \bar{\omega}\left(d_{2}\right)+$ $+\bar{\omega}\left(d_{1}\right) O_{1}+O_{1} \subset O$. В силу равномерной непрерывности отображения $\bar{\omega}$, в алгебре $D$ существует окрестность нуля $U$ такая, что при $d-d^{\prime} \in U$ справедливо $\bar{\omega}(d)-\bar{\omega}\left(d^{\prime}\right) \in O_{1}$. Теперь окрестность нуля $U$ определяет (в силу непрерывности умножения в $D$ ) закругленную окрестность нуля $U_{1}$ алгебры $D$ такую, что $U_{1} \subset U$ и $U_{1}^{2}+U_{1} d_{2}+d_{1} U_{1} \subset U$, а окрестность нуля $U_{1}$, в свою очередь, определяет (ввиду всюду плотности подалгебры

* Аналогичный результат в частном случае сформулирован в $\left[{ }^{4}\right]$ с. 346 . 
$E$ в $D)$ элементы $e_{1}, e_{2} \in E$ такие, что $e_{1}-d_{1} \in U_{1}$ и $e_{2}-d_{2} \in U_{1}$. Поскольку

$$
d_{1} d_{2}-e_{1} e_{2}=\left(e_{1}-d_{1}\right)\left(d_{2}-e_{2}\right)+\left(d_{1}-e_{1}\right) d_{2}+d_{1}\left(d_{R}-e_{2}\right),
$$

то $d_{1} d_{2}-e_{1} e_{2} \in U$. Поэтому по равенству

$$
\begin{gathered}
\left.\bar{\omega}\left(d_{1}\right) \bar{\omega}\left(d_{2}\right)-\bar{\omega}\left(d_{1} d_{2}\right)=\overline{(\omega}\left(e_{1}\right)-\bar{\omega}\left(d_{1}\right)\right)\left(\bar{\omega}\left(d_{2}\right)-\bar{\omega}\left(e_{2}\right)\right)+ \\
+\left(\bar{\omega}\left(d_{1}\right)-\bar{\omega}\left(e_{1}\right)\right) \bar{\omega}\left(d_{2}\right)+\bar{\omega}\left(d_{1}\right)\left(\bar{\omega}\left(d_{2}\right)-\bar{\omega}\left(e_{2}\right)\right)-\left(\bar{\omega}\left(d_{1} d_{2}\right)-\bar{\omega}\left(e_{1} e_{2}\right)\right)
\end{gathered}
$$

справедливо $\bar{\omega}\left(d_{1}\right) \bar{\omega}\left(d_{2}\right)-\bar{\omega}\left(d_{1} d_{2}\right) \in O$. В силу отделимости алгебры $H$ отсюда вытекает равенство $\bar{\omega}\left(d_{1}\right) \omega\left(d_{2}\right)=\bar{\omega}\left(d_{1} d_{2}\right)$. Итак, $\bar{\omega} \in$ hom $(D, H)$.

Так как алгебра $H$ отделима и подалгебра $E$ всюду плотна в $D$, то $\varrho_{1}\left|E \neq \varrho_{2}\right| E$ для двух различных гомоморфизмов $\varrho_{1}, \varrho_{2} \in \operatorname{hom}(D, H)$. Поэтому $\mu$ является взаимно однозначным отображением пространства $\operatorname{hom}(D, H)$ на $\operatorname{hom}(E, H)$.

Пусть теперь $\varrho_{0} \in \operatorname{hom}(D, H)$ и $O\left(\omega_{0}\right)$ - любая окрестность элемента $\omega_{0}=\varrho_{0} \mid E$ в топологии пространства $\operatorname{hom}(E, H)$. Нетрудно заметить, что множество $\mu^{-1}\left(O\left(\omega_{0}\right)\right)=\left\{\varrho \in \operatorname{hom}(D, H): \varrho \mid E \in O\left(\omega_{0}\right)\right\}$ является окрестностью гомоморфизма @о в топологии пространства $\operatorname{hom}(D, H)$. Значит, отображение $\mu$ непрерывно в любой точке $\varrho_{0} \Subset$ $\in \operatorname{hom}(D, H)$.

Остается показать непрерывность отображения $\mu^{-1}$ на $\operatorname{hom}(E, H)$, при условии, что пространство $\operatorname{hom}(E, H)$ локально равностепенно непрерывно. Для этого пусть $\omega_{1} \in \operatorname{hom}(E, H)$ и $\varrho_{1} \in \operatorname{hom}(D, H)$ такой гомоморфизм, что $\varrho_{1} \mid E=\omega_{1}$. Пусть далее $O\left(\varrho_{1}\right)-$ любая окрестность гомоморфизма @1 в топологии пространства $\operatorname{hom}(D, H)$. Тогда существуют окрестности нуля $V_{1}, V_{2}, \ldots, V_{m}$ алгебры $H$ и элементы $d_{1}, d_{2}, \ldots, d_{m} \in D$ такие, что

$$
\bigcap_{k=1}^{m}\left\{\varrho \in \operatorname{hom}(D ; H):\left(\varrho-\varrho_{1}\right)\left(d_{k}\right) \in V_{k}\right\} \subseteq O\left(\varrho_{1}\right) .
$$

Теперь окрестности $V_{1}, V_{2}, \ldots, V_{m}$ определяют открытые окрестности нуля $U_{1}, U_{2}, \ldots, U_{m}$ алгебры $H$ такие, что $U_{k}-U_{k} \subset V_{k}$ для всех $k \in \mathbf{N}_{m}$. Так как пространство $\operatorname{hom}(E, H)$ локально равностепенно непрерывно, то в $\operatorname{hom}(E, H)$ существует равностепенно непрерывная окрестность $O_{0}\left(\omega_{1}\right)$ элемента $\omega_{1}$.

Далее покажем, что множество $K=\mu^{-1}\left(O_{0}\left(\omega_{1}\right)\right)$ является равностепенно непрерывным в пространстве $\operatorname{hom}(D, H)$. Пусть $W_{0}$ - любая окрестность нуля алгебры $H$. Тогда найдутся замкнутая окрестность нуля $\mathbb{W}_{1} \subset W_{0}$ и открытая окрестноеть нуля $T$ в топологии подалгебры $E$ такие, что $\omega(T) \subset W_{1}$ при всех $\omega \in O_{0}\left(\omega_{1}\right)$. Пусть $T=S \cap E$, где $S-$ открытая окрестность нуля алгебры $D$, и $O\left(d_{0}\right)$ - любая окрестность элемента $d_{0} \in S$ в алгебре $D$. Тогда $O\left(d_{0}\right) \cap S$ является также окрестностью элемента $d_{0}$ в топологии алгебры $D$ и $O\left(d_{0}\right) \cap T=\left(O\left(d_{0}\right) \cap S\right) \cap E$. Поскольку подалгебра $E$ всюду плотна в $D$, то $O\left(d_{0}\right) \cap T$ непусто. Следовательно, элемент $d_{0}$ принадлежит $\mathrm{cl}_{D} T$. Таким образом, справедливо $S \subset \mathrm{cl}_{D} T$, в силу чего $\mathrm{cl}_{D} T$ является окрестностью нуля алгебры $D$.

Пусть теперь @ любой элемент в $K$. Тогда, ввиду непрерывности гомоморфизма $\varrho$ и включения $T \subset E$ имеем $\varrho\left(\mathrm{cl}_{D} T\right) \subseteq \mathrm{cl}_{H}(\varrho(T))=$ $=\mathrm{cl}_{H}(\varrho \mid E(T)) \subset W_{1} \subset W_{0}$. Значит, $K$ является равностепенно непрерывным в точке $\Theta_{D}$. Следовательно. $K$ есть равностепенно непрерывное подмножество в $\operatorname{hom}(D, H)$. Поэтому в алгебре $D$ существуют окрестности $O\left(d_{k}\right)$ элементов $d_{h}$ такие, что $\varrho(g)-\varrho\left(d_{k}\right) \in-U_{k}$ при всех $\varrho \in K$ и $g \in O\left(d_{k}\right)$ с $k \in \mathbf{N}_{m}$. В силу этого для каждого $k \in \mathbf{N}_{m}$ существуют $m_{k} \in O\left(d_{k}\right) \cap E$ такие, что $\omega_{1}\left(m_{k}\right)-\varrho_{1}\left(d_{k}\right)=\varrho_{1}\left(m_{k}\right)-\varrho_{1}\left(d_{k}\right) \in-U_{k}$. 
Следовательно, $\quad P_{k}=-U_{k}+\varrho_{1}\left(d_{k}\right)-\omega_{1}\left(m_{k}\right)$ нуля алгебры $H$ при всех $k \in \mathbf{N}_{m}$. Пусть далее

$$
O_{1}\left(\omega_{1}\right)=\bigcap_{k=1}^{m}\left\{\omega \in \operatorname{hom}(E, H):\left(\omega-\omega_{1}\right)\left(m_{k}\right) \in P_{k}\right\}
$$

и $O_{2}\left(\omega_{1}\right)=O_{0}\left(\omega_{1}\right) \cap O_{1}\left(\omega_{1}\right)$. Тогда $O_{2}\left(\omega_{1}\right)$ есть окрестность гомоморфизма $\omega_{1}$ в топологии пространства $\operatorname{hom}(E, H)$. Теперь $\bar{\omega}=\mu^{-1}(\omega) \in K$ при $\omega \in O_{2}\left(\omega_{1}\right)$ и

$$
\begin{gathered}
\left(\bar{\omega}-\varrho_{1}\right)\left(d_{k}\right)=\left(\bar{\omega}\left(d_{k}\right)-\bar{\omega}\left(m_{k}\right)\right)+\left(\omega\left(m_{k}\right)-\omega_{1}\left(m_{k}\right)\right)+ \\
+\omega_{1}\left(m_{k}\right)-\varrho_{1}\left(d_{k}\right) \in U_{k}+P_{k}+\omega_{1}\left(m_{k}\right)-\varrho_{1}\left(d_{k}\right)=U_{k}-U_{k} \subset V_{k}
\end{gathered}
$$

для всех $k \in \mathbf{N}_{m}$. Следовательно, $\mu^{-1}$ является непрерывным отображением в любой точке $\omega_{1} \in \operatorname{hom}(E, H)$.

Доказательство теоремы 2. Пусть $\Lambda \in \operatorname{hom}(B, C)$. Тогда $\Lambda \mid A Z \in \operatorname{hom}(A Z, C)$ в силу условия (В). Поэтому по теореме 1 существует элемент $(\lambda, \varrho) \in S_{A Z c}$ такой, что $\Lambda \mid A Z=\Lambda[\lambda, \varrho]$. По предложению 1 гомоморфизм $\Lambda[\lambda, \varrho]$ продолжим до гомоморфизма $\overline{\Lambda[\lambda, \varrho]} \in \operatorname{hom}(B, C)$. Поскольку алгебра $C$ отделима, то $\Lambda=\overline{\Lambda[\lambda, \varrho]}$. Итак, гомоморфизм $\Lambda$ определяет элемент $(\lambda, \varrho) \in S_{A Z C}$ такой, что $\Lambda=\overline{\Lambda[\lambda, \varrho]}$.

Пусть далее $\varphi$ - отображение, определенное в теореме 1 , а $\mu$-отображение $\operatorname{hom}(B, C)$ на $\operatorname{hom}(A Z, C)$, определяемое равенством $\mu(\Lambda)=$ $=\Lambda \mid A Z$ при всех $\Lambda \in \operatorname{hom}(B, C)$. Так как $\varphi$ есть гомеоморфизм пространств $S_{A Z C}$ и $\operatorname{hom}(A Z, C)$, а $\mu-$ непрерывная биекция $\operatorname{hom}(B, C)$ на $\operatorname{hom}(A Z, C)$ (по предложению 1), то отображение $\Phi=\mu^{-1} \circ \varphi$ является биекцией $S_{A z C}$ на $\operatorname{hom}(B, C)$, обратное отображение которой непрерывно на $\operatorname{hom}(B, C)$. Если при этом выполнено и условие (г), то $\mu$ есть гомеоморфизм пространств $\operatorname{hom}(B, C)$ и $\operatorname{hom}(A Z, C)$. Поэтому в таком случае отображение $\Phi$ является гомеоморфизмом пространств $S_{A z C}$ и $\operatorname{hom}(B, C)$. Теорема 2 доказана.

Вдобавок к вышеупомянутым условиям (а) и (б) при $C=\mathbf{K}$ пусть выполнено условие

(д) для каждого $\varrho \in$ hom $Z$ существует непрерывный $A$-гомоморфизм $\Phi_{\rho}$ алгебры $B$ на $A$ такой, что $\Phi_{\rho}(z)=e_{A} \varrho(z)$ для всех $z \in Z$. Тогда при всех $(\lambda ; \varrho) \in H_{\text {AZK }}, n \in \mathbf{N}, a_{i} \in A$ и $z_{i} \in Z$ с $i \in \mathbf{N}_{n}$ справедливо

$$
\sum_{k=1}^{n} \lambda\left(a_{k}\right) \varrho\left(z_{k}\right)=\lambda\left(\sum_{k=1}^{n} a_{k} \varrho\left(z_{k}\right)\right)=\left(\lambda \cdot \Phi_{\rho}\right)\left(\sum_{k=1}^{n} a_{k} z_{k}\right) .
$$

Поэтому в данном случае $S_{A Z K}=H_{A Z K}$. - Учитывая это, из теоремы 2 вытекает

С л е д с т в и е 1. (ср. $\left[{ }^{5}\right]$, с. 2$)$. Пусть $A$ - отделимая топологическая К-алгебра с единицей, $B$ - топологическая $(A, \mathbf{K})$-алгебра с единицей ** и Z подалгебра алгебры В такая, что $e_{R} \in Z$. Если выполнены условия (а), (б) (при C=K), (в) $u$ (д), то каждый $\Lambda \in$ hom $B$ определяет $\lambda \in \operatorname{hom} A$ u $е$ hom $Z$ такие, что $\Lambda=\lambda \circ \Phi_{\rho} u \Lambda \rightarrow(\lambda, \mathrm{e})$ отображает hom $B$ взаимно однозначно на $H_{A Z K}$ -

Д ок а з а тель с т в о теоремы 3. Пусть $f$ - топологический изоморфизм, полученный при пополнении алгебры $B$. Тогда отображение $f^{*}$, определяемое равенством $f^{*}(\omega)=0 \circ f$ при всех $\omega \in \operatorname{hom}(f(B), C)$, является

\footnotetext{
** Так как по условию (д) каждый ғомоморфизм $\Lambda[\lambda, \varrho] \in \operatorname{hom} A Z$ продолжим до гомоморфизма $\overline{\Lambda[\lambda, g]}=\lambda_{\circ} \Phi_{\rho}$, то непрерывности умножения в алгебре $B$ не требуется.
} 
гомеоморфизмом пространств $\operatorname{hom}(f(B), C)$ и $\operatorname{hom}(B, C)$. Нетрудно заметить, что из локально равностепенной непрерывности пространства $\operatorname{hom}(A Z, C)$ вытекает локально равностепенная непрерывность пространства $\operatorname{hom}(B, C)$ (см. доказательство предложения 1$)$. Поэтому и пространство hom $(f(B), C)$ является локально равностепенно непрерывным.

Пусть теперь $\varphi-$ гомеоморфизм $S_{A z C}$ на $\operatorname{hom}(A Z, C)$, определенный в теореме 1 , а $\mu_{1}: \operatorname{hom}(B, C) \rightarrow \operatorname{hom}(A Z, C)$ и $\mu_{2}: \operatorname{hom}(\bar{B}, C) \rightarrow$ $\rightarrow \operatorname{hom}(f(B), C)$ - гомеоморфизмы, определяемые равенствами $\mu_{1}(\varrho)=$ $=\mathrm{Q} \mid A Z$ при всех $\mathrm{Q} \in \operatorname{hom}(B, C)$ и $\mu_{2}(\Lambda)=\Lambda \mid f(B)$ при всех $\Lambda \in \operatorname{hom}(\bar{B}, C)$. Тогда отображение $\Psi=\mu_{2}^{-1} \circ\left(f^{*}\right)^{-1} \circ \mu_{1}^{-1} \circ \varphi$ является гомеоморфизмом пространств $S_{A Z C}$ и hom $(\bar{B}, C)$.

\section{Л ИТЕ РАТ У РА}

1. Horváth, J. Topological Vector Spaces and Distributions. V. 1. Massachusetts, Addison-Wesley Reading, 1966.

2. Cochran, A. C., Keown, R., Williams, C. R. Pacif. J. Math., 34, 17-25 (1970).

3. Бурбаки H. Общая топология. Топологические группы. Чнсла и связанные с ними группы и пространства. М., «Наука», 1969.

4. Mallios, A. Lect. Notes in Math., 399. Berlin, Springer-Verlag, 1974, 342-377.

5. Abel, M. In: Abstracts. Colloquium on Topology, Eger, Aug. 9-13, 1983. Budapest, Janos Bolyai Math. Soc., 1983, 2.

Тартуский государственный университет

Поступила в редакцию

7/IV 1986

A. $K O K K$.

\section{TOPOLOOGILISTE MOODULALGEBRATE HOMOMORFISMIDE KIRJELDUS}

On antud ühikuga topoloogilise moodulalgebra kõigi mittetriviaalsete pidevate homomorfismide kirjeldus.

\section{A. $K O K K$}

\section{THE REPRESENTATION OF HOMOMORPHISMS OF TOPOLOGICAL MODULE-ALGEBRAS}

Let $\mathbf{K}$ be the field of real or complex numbers; let $A$ be a topological $\mathbf{K}$-algebra with the unit $e_{A}$, and let $C$ be a topological $\mathbf{K}$-algebra with a jointly continuous multiplication. Let $B$ be a topological $(A, \mathbf{K})$-algebra with the unit $e_{B}$ (that is a topological K-algebra with a unit which at the same time is a $A$-bimodule with separately continuous module multiplications and in which the conditions $a\left(b_{1} b_{2}\right)=\left(a b_{1}\right) b_{2},\left(b_{1} b_{2}\right) a=$ $=b_{1}\left(b_{2} a\right), b_{1}\left(a b_{2}\right)=\left(b_{1} a\right) b_{2}, e_{A} b=b=b e_{A}$ and $a e_{B}=e_{B} a$ hold for each $a \in A$ and $\left.b_{1}, b_{2} \in B\right)$, let $Z$ be a subalgebra of $B$ with the unit $e_{B}$ and hom $(B, C)$ be the set of all non-zero continuous $\mathbf{K}$-homomorphisms of $B$ into $C$, endowed with the topology of simple convergence in $B$.

If the conditions

(a) $a z=z a$ for each $a \in A$ and $z \in Z$, and

(b) the A-submodule $A Z$ of $B$, generated by $Z$, is dense in $B$

are satisfied then the spaces hom $(A Z, C)$, hom $(B, C)$ and hom $(\bar{B}, C)$ (where $\bar{B}$ stands

for the completion of $B$ ) are characterized, 